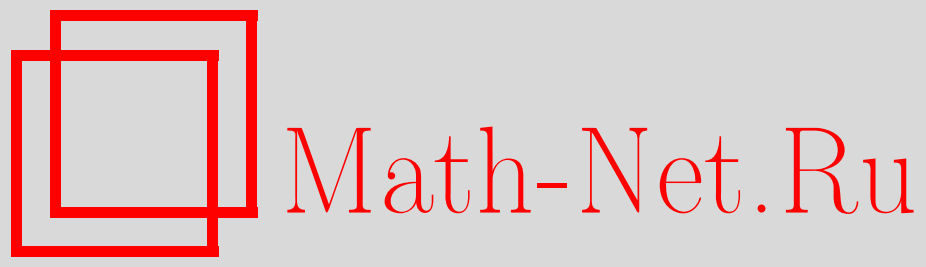

А. Д. Агальцов, Теорема характеризации для обобщенного преобразования Радона, возникающего в одной модели математической экономики, Функи. анализ и его прил., 2015, том 49, выпуск 3, 57-60

DOI: https://doi.org/10.4213/faa3201

Использование Общероссийского математического портала MathNet.Ru подразумевает, что вы прочитали и согласны с пользовательским соглашением

http://www . mathnet.ru/rus/agreement

Параметры загрузки:

IP : 3.89 .185 .249

26 апреля 2023 г., 13:30:12

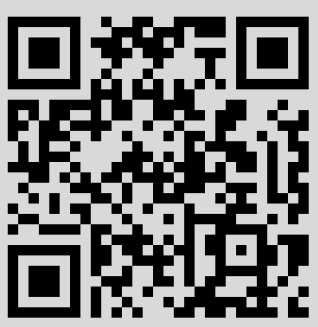




\title{
Теорема характеризации для обобщенного преобразования Радона, возникающего в одной модели математической экономики*
}

\author{
(c) 2015. А. Д. АгАльцОв
}

1. Введение. В работе [5] была предложена модель производства в чистой отрасли, в которой учитывается эффект взаимного замещения производственных факторов на микроуровне. Функция прибыли в этой модели имеет вид

$$
\left(\Pi_{q} \mu\right)\left(p_{0}, p\right)=\int_{\mathbb{R}_{+}^{n}}\left(p_{0}-q\left(p_{1} x_{1}, \ldots, p_{n} x_{n}\right)\right)+\mu(d x),
$$

где $p_{0}$ - цена выпускаемой продукции, $p=\left(p_{1}, \ldots, p_{n}\right)$ - вектор цен на производственные факторы, $\mu$ - распределение мощностей по технологиям, $q: \mathbb{R}_{+}^{n} \rightarrow$ $\mathbb{R}_{+}$- функция себестоимости выпускаемой продукции, $a_{+}=\max (a, 0), x=$ $\left(x_{1}, \ldots, x_{n}\right), \mathbb{R}_{+}^{n}=\left\{\left(x_{1}, \ldots, x_{n}\right) \in \mathbb{R}^{n}, x_{i} \geqslant 0\right\}$. Преобразование (1) сводится повторным дифференцированием по $p_{0}$ к обобщенному преобразованию Радона меры $\mu$, которое определяется как производная в смысле теории распределений:

$$
\left.R_{q} \mu\left(p_{0}, p\right)=\frac{\partial}{\partial p_{0}} \mu\left\{x \in \mathbb{R}_{+}^{n}: q\left(p_{1} x_{1}, \ldots, p_{n} x_{n}\right) \leqslant p_{0}\right)\right\}, \quad x=\left(x_{1}, \ldots, x_{n}\right) .
$$

Указанная связь делает задачи характеризации для преобразования (1) и для обобщенного преобразования Радона практически эквивалентными. Мы приведем теорему характеризации для преобразования (1).

В работе [4] была получена теорема характеризации для интегрального преобразования (1) в случае линейной функции $q$, что соответствует отсутствию эффекта замещения производственных факторов. В работе [1] эта теорема была обобщена на случай преобразований вида (1) с функцией $q=q_{\alpha}$ вида $q_{\alpha}(x)=$ $\left(x_{1}^{\alpha}+\cdots+x_{n}^{\alpha}\right)^{1 / \alpha}, x=\left(x_{1}, \ldots, x_{n}\right), 0<\alpha \leqslant 1$, что с экономической точки зрения отвечает производственным системам с постоянной эластичностью замещения.

Основным результатом этой работы является следующее обобщение указанных теорем на случай произвольных положительно однородных функций себестоимости $q$. Пусть $c \in \operatorname{int} \mathbb{R}_{+}^{n}=\left\{\left(x_{1}, \ldots, x_{n}\right) \in \mathbb{R}^{n}, x_{i}>0\right\}$, а $M$ и $M_{c}^{-1}$ обозначают прямое и обратное преобразования Меллина:

$$
M \varphi(z)=\int_{\mathbb{R}_{+}^{n}} x^{z-I} \varphi(x) d x, \quad M_{c}^{-1} \psi(x)=(2 \pi i)^{-n} \int_{c+i \mathbb{R}^{n}} x^{-z} \psi(z) d z,
$$

где $x=\left(x_{1}, \ldots, x_{n}\right), z=\left(z_{1}, \ldots, z_{n}\right), I=(1, \ldots, 1), x^{z-I}=x_{1}^{z_{1}-1} \cdots x_{n}^{z_{n}-1}$, $x^{-z}=x_{1}^{-z_{1}} \cdots x_{n}^{-z_{n}}$ (всюду далее вектор, возведенный в векторную степень, понимается в этом смысле). 
Пусть $q: \mathbb{R}_{+}^{n} \rightarrow \mathbb{R}_{+}$такова, что значение $\left(M e^{-q}\right)(z)$ определено и отлично от нуля для почти всех $z \in c+i \mathbb{R}^{n}$, где $c \in \operatorname{int} \mathbb{R}_{+}^{n}$. Мы полагаем

$$
\rho_{q}(z)=\frac{\Gamma\left(z_{1}\right) \cdots \Gamma\left(z_{n}\right) \Gamma\left(2+z_{1}+\cdots+z_{n}\right)}{\left(M e^{-q}\right)(z)},
$$

где $z=\left(z_{1}, \ldots, z_{n}\right), z \in c+i \mathbb{R}^{n}$, а $\Gamma(\cdot)$ - гамма-функция Эйлера.

Мы будем называть функцию $g: \mathbb{R}_{+}^{n} \rightarrow \mathbb{R}$ вполне монотонной, если $g \in$ $C^{\infty}\left(\operatorname{int} \mathbb{R}_{+}^{n}\right)$ и $(-1)^{|\alpha|} \partial^{|\alpha|} g(p) / \partial p^{\alpha} \geqslant 0$ для всех $\alpha \in \mathbb{Z}_{+}^{n}, p \in \operatorname{int} \mathbb{R}_{+}^{n}$.

Tеорема. Пусть $q \in C\left(\mathbb{R}_{+}^{n}\right) \cap C^{1}\left(\operatorname{int} \mathbb{R}_{+}^{n}\right), q(x)>0$ nрu $x \in \operatorname{int} \mathbb{R}_{+}^{n}, q(\lambda x)=$ $\lambda q(x)$ nри $x \in \operatorname{int} \mathbb{R}_{+}^{n}, \lambda>0$, и пусть $\rho_{q}(z) \in L^{2} \cup L^{\infty}\left(c+i \mathbb{R}^{n}\right)$, где $c \in \operatorname{int} \mathbb{R}_{+}^{n}$. Пусть П: $\mathbb{R}_{+} \times \mathbb{R}_{+}^{n} \rightarrow \mathbb{R}_{+}$. Положим $f(p)=\Pi(1, p), g=M_{c}^{-1}\left(\rho_{q} M f\right)$.

При этих условиях функиия П представима в виде $\Pi=\Pi_{q} \mu$, где $\mu$ - неотричательная конечная борелевская мера на $\mathbb{R}_{+}^{n}$, такал, что $x^{-c}, x^{-2 c} \in L^{1}(\mu)$, a $\Pi_{q} \mu$ определена формулой (1), тогда и только тогда, когда

(i) $p^{c-I} f(p), p^{2 c-I}|f(p)|^{2} \in L^{1}\left(\mathbb{R}_{+}^{n}\right)$;

(ii) $p^{c-I} g(p), p^{2 c-I} g(p) \in L^{1}\left(\mathbb{R}_{+}^{n}\right), g$ вполне монотонна и $g(+0)<\infty$;

(iii) $\Pi\left(\lambda p_{0}, \lambda p\right)=\lambda \Pi\left(p_{0}, p\right)$ npu $\lambda>0, p_{0}>0, p \in \operatorname{int} \mathbb{R}_{+}^{n}$.

\section{2. Вспомогательные утверждения.}

Лемма 1. Пусть $q \in C\left(\mathbb{R}_{+}^{n}\right) \cap C^{1}\left(\operatorname{int} \mathbb{R}_{+}^{n}\right), q(x)>0$ npu $x \in \operatorname{int} \mathbb{R}_{+}^{n} u$ $q(\lambda x)=\lambda q(x)$ для всех $x \in \operatorname{int} \mathbb{R}_{+}^{n}, \lambda>0$. Пусть $t^{c_{1}+\cdots+c_{n}-1} h(t) \in L^{1}(0,+\infty)$ при некотором $c=\left(c_{1}, \ldots, c_{n}\right) \in \operatorname{int} \mathbb{R}_{+}^{n}$. Тогда при $z \in c+i \mathbb{R}^{n}$ справедливо равенство

$$
\int_{\mathbb{R}_{+}^{n}} p^{z-I} h\left(q\left(p_{1} x_{1}, \ldots, p_{n} x_{n}\right)\right) d p=x^{-z} \frac{\left(M e^{-q}\right)(z)(M h)\left(z_{1}+\cdots+z_{n}\right)}{\Gamma\left(z_{1}+\cdots+z_{n}\right)} .
$$

Доказательство этой леммы см. в [2, лемма 1, с. 6].

Лемма 2. Пусть $q \in C\left(\mathbb{R}_{+}^{n}\right) \cap C^{1}\left(\operatorname{int} \mathbb{R}_{+}^{n}\right), q(x)>0$ nрu $x \in \operatorname{int} \mathbb{R}_{+}^{n}$, $q(\lambda x)=\lambda q(x)$ для всех $x \in \operatorname{int} \mathbb{R}_{+}^{n}, \lambda>0$. Пусть $t^{c_{1}+\cdots+c_{n}-1} h(t) \in L^{1}(0,+\infty)$ $u t^{2 c_{1}+\cdots+2 c_{n}-1}|h(t)|^{2} \in L^{1}(0,+\infty)$ при некотором $c=\left(c_{1}, \ldots, c_{n}\right) \in \operatorname{int} \mathbb{R}_{+}^{n}, u$ пусть $h$ ограничена на $[0,+\infty)$. Пусть $\mu$ - неотрицательная конечная борелевская мера на $\mathbb{R}_{+}^{n}$, такая, что $x^{-c}, x^{-2 c} \in L^{1}(\mu)$. Тогда $p^{c-I} R_{q}^{h} \mu(p) \in L^{1}\left(\mathbb{R}_{+}^{n}\right)$ и $p^{2 c-I}\left|R_{q}^{h} \mu(p)\right|^{2} \in L^{1}\left(\mathbb{R}_{+}^{n}\right)$ (как функиии аргумента $\left.p\right)$, где

$$
R_{q}^{h} \mu(p)=\int_{\mathbb{R}_{+}^{n}} h\left(q\left(p_{1} x_{1}, \ldots, p_{n} x_{n}\right)\right) \mu(d x), \quad p=\left(p_{1}, \ldots, p_{n}\right) .
$$

Доказательство. В силу ограниченности функции $h$ и конечности меры $\mu$ функция $R_{q}^{h} \mu(p)$ определена при $p \in \mathbb{R}_{+}^{n}$. По теореме Фубини и с учетом леммы 1 можно записать

$$
\int_{\mathbb{R}_{+}^{n}} p^{c-I}\left|\left(R_{q}^{h} \mu\right)(p)\right| d p \leqslant \int_{\mathbb{R}_{+}^{n}} x^{-c} \mu(d x) \frac{\left(M e^{-q}\right)(c)(M|h|)\left(c_{1}+\cdots+c_{n}\right)}{\Gamma\left(c_{1}+\cdots+c_{n}\right)} .
$$

В условиях настоящей леммы выражение справа конечно; поэтому $p^{c-I} R_{q}^{h} \mu(p) \in$ $L^{1}\left(\mathbb{R}_{+}^{n}\right)$. Далее, используя неравенство Йенсена, запишем

$$
\left|\left(R_{q}^{h} \mu\right)(p)\right|^{2} \leqslant \mu\left(\mathbb{R}_{+}^{n}\right) \int_{\mathbb{R}_{+}^{n}}\left|h\left(q\left(p_{1} x_{1}, \ldots, p_{n} x_{n}\right)\right)\right|^{2} \mu(d x)=\mu\left(\mathbb{R}_{+}^{n}\right)\left(R_{q}^{|h|^{2}} \mu\right)(p) .
$$


С учетом этого неравенства и леммы 1 получим следующее неравенство:

$\int_{\mathbb{R}_{+}^{n}} p^{2 c-I}\left|R_{q}^{h} \mu(p)\right|^{2} d p \leqslant \mu\left(\mathbb{R}_{+}^{n}\right) \int_{\mathbb{R}_{+}^{n}} x^{-2 c} \mu(d x) \frac{\left(M e^{-q}\right)(2 c)\left(M|h|^{2}\right)\left(2 c_{1}+\cdots+2 c_{n}\right)}{\Gamma\left(2 c_{1}+\cdots+2 c_{n}\right)}$.

Из конечности последнего выражения вытекает, что $p^{2 c-I}\left|R_{q}^{h} \mu(p)\right|^{2} \in L^{1}\left(\mathbb{R}_{+}^{n}\right)$.

Лемма 3. Пусть $q_{k} \in C\left(\mathbb{R}_{+}^{n}\right) \cap C^{1}\left(\operatorname{int} \mathbb{R}_{+}^{n}\right), q_{k}(x)>0$ npu $x \in \operatorname{int} \mathbb{R}_{+}^{n}, q_{k}(\lambda x)=$ $\lambda q_{k}(x)$ для всех $x \in \operatorname{int} \mathbb{R}_{+}^{n}, \lambda>0, k=1,2$. Пусть $t^{c_{1}+\cdots+c_{n}-1} h_{k}(t) \in L^{1}(0,+\infty)$ $u t^{2 c_{1}+\cdots+2 c_{n}-1}\left|h_{k}(t)\right|^{2} \in L^{1}(0,+\infty)$ nри некотором $c=\left(c_{1}, \ldots, c_{n}\right) \in$ int $\mathbb{R}_{+}^{n}$, $h_{k}$ ограниченъ на $[0,+\infty), k=1,2$. Пусть функиия

$$
\sigma_{q_{1}, q_{2}}^{h_{1}, h_{2}}(z)=\frac{\left(M e^{-q_{1}}\right)(z)\left(M h_{1}\right)\left(z_{1}+\cdots+z_{n}\right)}{\left(M e^{-q_{2}}\right)(z)\left(M h_{2}\right)\left(z_{1}+\cdots+z_{n}\right)}, \quad z=\left(z_{1}, \ldots, z_{n}\right),
$$

определена при почти всех $z \in c+i \mathbb{R}^{n}$. Пусть $\mu$-неотрицательная конечная борелевская мера на $\mathbb{R}_{+}^{n}$, такая, что $x^{-c}, x^{-2 c} \in L^{1}(\mu)$. Пусть оператор $R_{q}^{h}$ определен бормулой (2). Тогда справедливы следующие утвержсения.

(A) Имеет место формула $R_{q_{1}}^{h_{1}} \mu=M_{c}^{-1}\left(\sigma_{q_{1}, q_{2}}^{h_{1}, h_{2}} M R_{q_{2}}^{h_{2}} \mu\right)$.

(B) Пусть $\sigma_{q_{1}, q_{2}}^{h_{1}, h_{2}} \in L^{2}\left(c+i \mathbb{R}^{n}\right) \cup L^{\infty}\left(c+i \mathbb{R}^{n}\right)$ u $\sigma_{q_{1}, q_{2}}^{h_{1}, h_{2}}(z) \neq 0$ для почти всех $z \in c+i \mathbb{R}^{n}$. Пусть $p^{c-I} f(p), p^{2 c-I}|f(p)|^{2} \in L^{1}\left(\mathbb{R}_{+}^{n}\right)$, и пусть выполнено равенство $R_{q_{1}}^{h_{1}} \mu=M_{c}^{-1}\left(\sigma_{q_{1}, q_{2}}^{h_{1}, h_{2}} M f\right)$. Тогда $f=R_{q_{2}}^{h_{2}} \mu$.

Доказательство. (А) Пользуясь леммой 1 и теоремой Фубини, получим при $z \in c+i \mathbb{R}^{n}$ следующее равенство:

$$
\left(M R_{q_{k}}^{h_{k}} \mu\right)(z)=\int_{\mathbb{R}_{+}^{n}} x^{-z} \mu(d x) \frac{\left(M e^{-q_{k}}\right)(z)\left(M h_{k}\right)\left(z_{1}+\cdots+z_{n}\right)}{\Gamma\left(z_{1}+\cdots+z_{n}\right)}, \quad k=1,2 .
$$

Отсюда вытекает, что

$$
\left(M R_{q_{1}}^{h_{1}} \mu\right)(z)=\sigma_{q_{1}, q_{2}}^{h_{1}, h_{2}}(z)\left(M R_{q_{2}}^{h_{2}} \mu\right)(z) .
$$

Из леммы 2 следует, что $p^{c-I} R_{q_{1}}^{h_{1}} \mu(p), p^{2 c-I}\left|R_{q_{1}}^{h_{1}} \mu(p)\right|^{2} \in L^{1}\left(\mathbb{R}_{+}^{n}\right)$. Поэтому к равенству (4) можно применить обратное преобразование Меллина, так что слева получится $R_{q_{1}}^{h_{1}} \mu$.

(В) По условию и в силу доказанного п. (А) имеем

$$
M_{c}^{-1}\left(\sigma_{q_{1}, q_{2}}^{h_{1}, h_{2}} M f\right)=M_{c}^{-1}\left(\sigma_{q_{1}, q_{2}}^{h_{1}, h_{2}} M R_{q_{2}}^{h_{2}} \mu\right),
$$

так как оба выражения в нем равны $R_{q_{1}}^{h_{1}} \mu$. Согласно условию и лемме $2, p^{c-I} f(p)$, $p^{2 c-I}|f(p)|^{2}, p^{c-I} R_{q_{2}}^{h_{2}} \mu(p), p^{2 c-I}\left|R_{q_{2}}^{h_{2}} \mu(p)\right|^{2} \in L^{1}\left(\mathbb{R}_{+}^{n}\right)$. Поэтому $M f, M R_{q_{2}}^{h_{2}} \mu \in$ $L^{2}\left(c+i \mathbb{R}^{n}\right)$. Так как $\sigma_{q_{1}, q_{2}}^{h_{1}, h_{2}} \in L^{2}\left(c+i \mathbb{R}^{n}\right) \cup L^{\infty}\left(c+i \mathbb{R}^{n}\right)$, то $\sigma_{q_{1}, q_{2}}^{h_{1}, h_{2}} M f, \sigma_{q_{1}, q_{2}}^{h_{1}, h_{2}} M R_{q_{2}}^{h_{2}} \in$ $L^{2}\left(c+i \mathbb{R}^{n}\right)$. Наконец, по лемме $2 p^{c-I} R_{q_{1}}^{h_{1}} \mu(p), p^{2 c-I}\left|R_{q_{1}}^{h_{1}} \mu(p)\right|^{2} \in L^{1}\left(\mathbb{R}_{+}^{n}\right)$. Поэтому можно сначала применить к равенству (5) оператор $M$, потом поделить полученное равенство на $\sigma_{q_{1}, q_{2}}^{h_{1}, h_{2}}$, а затем применить к новому равенству оператор $M_{c}^{-1}$. В итоге мы придем к равенству $f=R_{q_{2}}^{h_{2}} \mu$.

3. Доказательство теоремы. Необходимость. Пусть $\Pi\left(p_{0}, p\right)=\left(\Pi_{q} \mu\right)\left(p_{0}, p\right)$. Свойство $\Pi\left(\lambda p_{0}, \lambda p\right)=\lambda \Pi\left(p_{0}, p\right)$ следует из формулы $(1)$.

Справедливо равенство $f=R_{q}^{h_{\Pi}} \mu$, где $h_{\Pi}(t)=(1-t)_{+}$и оператор $R_{q}^{h_{\Pi}}$ определен формулой (2). Кроме того, заметим, что $\rho_{q}=\sigma_{q_{L}, q}^{h_{L}, h_{\Pi}}$, где $h_{L}(t)=\exp (-t)$, 
$h_{\Pi}(t)=(1-t)_{+}, q_{L}(x)=x_{1}+\cdots+x_{n}$, а функция $\sigma_{q_{L}, q}^{h_{L}, h_{\Pi}}$ определена формулой (3). По лемме 2 имеем $p^{c-I} f(p), p^{2 c-I}|f(p)|^{2} \in L^{1}\left(\mathbb{R}_{+}^{n}\right)$. По лемме $3(\mathrm{~A})$ имеем $L \mu=M_{c}^{-1}\left(\rho_{q} M f\right) \equiv g$, где $L-$ многомерное преобразование Лапласа. Отсюда в силу теоремы характеризации для преобразования Лапласа (см. [3, теорема 4.2 .1 , с. 87]) вытекает, что функция $g$ вполне монотонна и $g(+0)=\mu\left(\mathbb{R}_{+}^{n}\right)<\infty$.

Из равенства $g=L \mu$ с учетом леммы 1 следует равенство

$$
\int_{\mathbb{R}_{+}^{n}} p^{c-I} g(p) d p=\int_{\mathbb{R}_{+}^{n}} x^{-c} \mu(d x) \Gamma\left(c_{1}\right) \cdots \Gamma\left(c_{n}\right) .
$$

Отсюда в силу $x^{-c} \in L^{1}(\mu)$ вытекает, что $p^{c-I} g(p) \in L^{1}\left(\mathbb{R}_{+}^{n}\right)$. Заменяя в равенстве (6) $p^{c-I}$ на $p^{2 c-I}$, получим, учитывая $x^{-2 c} \in L^{1}(\mu)$, что $p^{2 c-I} g(p) \in L^{1}\left(\mathbb{R}_{+}^{n}\right)$.

Достаточность. По условию функция $g=M_{c}^{-1}\left(\rho_{q} M f\right)$ вполне монотонна и $g(+0)<\infty$. По теореме характеризации для преобразования Лапласа (см. $[3$, теорема 4.2 .1 , с. 87]) найдется такая неотрицательная конечная борелевская мера $\mu$ на $\mathbb{R}_{+}^{n}$, что $g=L \mu$.

Так как по условию $p^{c-I} g(p) \in L^{1}\left(\mathbb{R}_{+}^{n}\right)$, то из равенства (6) следует, что $x^{-c} \in L^{1}(\mu)$. Заменяя в этом равенстве $p^{c-I}$ на $p^{2 c-I}$ и пользуясь тем, что $p^{2 c-I} g(p) \in L^{1}\left(\mathbb{R}_{+}^{n}\right)$, получим, что $x^{-2 c} \in L^{1}(\mu)$. Наконец, из равенства $g \equiv$ $M_{c}^{-1}\left(\rho_{q} M f\right)=L \mu$ в силу леммы $3(\mathrm{~B})$ следует, что $f(p)=\left(\Pi_{q} \mu\right)(1, p)$.

Из соотношения $\Pi(1, p) \equiv f(p)=\left(\Pi_{q} \mu\right)(1, p)$ в силу свойства $\Pi\left(\lambda p_{0}, \lambda p\right)=$ $\lambda \Pi\left(p_{0}, p\right)$ вытекает, что $\Pi\left(p_{0}, p\right)=\left(\Pi_{q} \mu\right)\left(p_{0}, p\right)$. Теорема доказана.

Замечание. Приведенная схема доказательства теоремы характеризации для функции прибыли (1) непосредственно переносится на случай произвольных операторов вида (2), например, на случай преобразования Фантаппье, которому соответствует выбор $q\left(x_{1}, \ldots, x_{n}\right)=x_{1}+\cdots+x_{n}, h(t)=(1+t)^{-1}$.

\section{ЛиТЕРАТУРА}

[1] А. Д. Агальцов, Труды МФТИ, 5:4 (20) (2013), 48-61. [2] А. Д. Агальцов, Труды МФТИ, 6:2 (22) (2014), 3-14. [3] S. Bochner, Harmonic Analysis and the Theory of Probability, University of California Press, Berkeley and Los Angeles, 1955. [4] G. M. Henkin, A. A. Shananin, in: Transl. Math. Monographs, vol. 81, Amer. Math. Soc., Providence, RI, 1990, 189-223. [5] А. А. Шананин, Матем. моделирование, 9:9 (1997), 117-127.

Московский государственный университет им. М. В. Ломоносова, факультет вычислительной математики и кибернетики CMAP, Ecole Polytechnique, France e-mail: agalets@gmail.com

УДК 519.2

\section{Замечания о квантовых марковских состояниях}

(C) 2015. З. И. БЕЖАЕВА, В. И. ОСЕЛЕДЕЦ

Посвящается А. М. Вершику по случаю его 80-летия

Введение. В настоящей заметке дается определение квантового марковского состояния на квазилокальной $C^{*}$-алгебре $\Omega_{d}=\bigotimes_{k \in N}\left(M_{d}\right)_{k}$, где $M_{d}$ есть $C^{*}$-алгебра комплексных матриц размера $d \times d$. 